29 Januar 1830.

Glinenoi. Vorposten auf der Kirgisischen Steppe. Breite $=48^{\circ} 56^{\prime} 47^{\prime \prime}$. Länge durch Chronometer ohngefähr $67^{\circ} 22^{\prime}$.

\begin{tabular}{|c|c|c|}
\hline $\begin{array}{c}\text { Kessels } 1259 \\
\text { h } 19^{\prime} 58^{\prime \prime}\end{array}$ & $\begin{array}{r}\text { Sonne - } \\
63^{\circ} 39^{\prime}\end{array}$ & $\begin{array}{l}\text { Mond } \\
9^{\prime} 15^{\prime \prime}\end{array}$ \\
\hline 2258 & 40 & 035 \\
\hline 2440 & 41 & 120 \\
\hline $\begin{array}{ll}267\end{array}$ & 41 & 150 \\
\hline 2736 & 42 & 210 \\
\hline 290 & 42 & 255 \\
\hline 3030 & 43 & 320 \\
\hline 324 & 43 & 345 \\
\hline 3320 & 44 & 75 \\
\hline 3431 & 43 & 345 \\
\hline
\end{tabular}

Red. auf M.Z. $=+$\begin{tabular}{lll}
0226,7 Indexfehler $=+3 \quad 14,2$ \\
\hline
\end{tabular}

M. Z. Glin. $=\overline{3^{h} 50^{\prime} 31^{\prime \prime 1}} \quad \overline{63^{\circ} 45^{\prime} 32^{\prime \prime 2}}$

Temperatur $=-17^{\circ}, 4 \mathrm{R}$.

Barom. $=775^{\mathrm{mm}}, 0+8^{\circ}, 0 \mathrm{R}$.

Da hirr der Indexfehler mit beiden rothen Blendgläsern bestimmt ist, die Distanzen aber blofs mit dem einen genommen sind, so findet die oben erwähnte Unsicherheit auch hier statt. Observ. $H$.

\section{Januar 1830.}

Der eben angeführte Ort.

\begin{tabular}{|c|c|}
\hline $\begin{array}{c}\text { Kessels } 1259 \\
\text { h } \\
6^{\prime} 36^{\prime} 25,5\end{array}$ & $\begin{array}{c}\text { Mond - Venus. } \\
25^{\circ} 9^{\prime} 10^{\prime \prime}\end{array}$ \\
\hline 3959,0 & 1015 \\
\hline 4134 & 1045 \\
\hline 4224 & 1110 \\
\hline 4510 & 1240 \\
\hline 4728 & 1335 \\
\hline 484 & 1410 \\
\hline
\end{tabular}

$$
\begin{aligned}
& \begin{array}{llll}
6 & 50 & 37
\end{array} \\
& 52 \quad 25 \\
& \text { Miltel }=\frac{5343}{6^{\mathrm{h}} 45^{\prime} 46^{\prime \prime} 95} \quad \frac{160}{25^{\circ} 12^{\prime} 46^{\prime \prime} 5}
\end{aligned}
$$

Red, auf M.Z. $=+2226,14$ Indexfehler $=+3 \quad 2,5$

M. Z. Glin. $=\overline{7^{\mathrm{h}}} \overline{8^{\prime} 13^{\prime \prime} 09} \quad \overline{25^{\mathrm{o}} 15^{\prime} 49^{\prime} 0}$

Temperatur kurz vor der Beobachtung $=-19^{\circ}, 0 \mathrm{R}$.

$$
\begin{aligned}
& \text { kur nach selbiger } \\
& =-20^{\prime} 0 \mathrm{R} \text {. } \\
& \text { Barom. }=775^{\mathrm{mm}, 9}+11^{\circ}, 8 \mathrm{R} \text {. }
\end{aligned}
$$

Der Indexfehler und die Distanzen sind ohne Blendglas gemessen. Bei den Distanzen ist zu bemerken, dafs die Mitte des beleuchteten Theils der Venus, welcher etwas weniger als dic Hälfte war, mit dem Rande des Mondes in Berührung gebracht wurde. Beobachter Lieutenant Due.

$$
\begin{aligned}
& \text { 30. Januar } 1830 . \\
& \text { Derselbe Ort. } \\
& \text { Kessels } 1259 \text { Mond - Aldebaran. } \\
& 7^{\text {h } 39^{\prime} 28^{\prime \prime}} \quad 37^{\circ} 42^{\prime} 40^{\prime \prime} \\
& 4229 \quad 4120 \\
& 4353 \quad 40 \quad 50 \\
& \begin{array}{llll}
45 \quad 2 & 40 \quad 15
\end{array} \\
& 4646 \quad 3940 \\
& 4821 \quad 390 \\
& \begin{array}{lll}
4939 & 38 \quad 15
\end{array} \\
& \begin{array}{llll}
51 & 4 & 3745
\end{array} \\
& 5431,5 \quad 36 \quad 0 \\
& \text { Mittel }=\frac{5645}{7^{\mathrm{h}} 47^{\prime} 47^{\prime \prime} 85} \quad \frac{355}{37^{\circ} 39^{\prime} 5}
\end{aligned}
$$

Red. auf M.Z. $=+2229,99$ Indexfehler $=+317,5$

$$
\begin{aligned}
\text { M. Z. Glin. } & =\overline{8^{\mathrm{h}} 10^{\prime} 17^{\prime \prime} 84} \quad \frac{37^{\circ} 42^{\prime} 22^{\prime \prime}, 5}{} \\
& \text { Temperatur }=-19^{\circ}, 0 \mathrm{R} . \\
& \text { Barom. }=771^{\mathrm{mm}}, 3+10^{\circ}, 9 \mathrm{R} .
\end{aligned}
$$

Der Indexfehler und die Distanzen wurden ohne Blendg!as bestimmt. Beobachter $\boldsymbol{H}$.

Hansteen.

Sternbedeckungen vom Monde, und Jupiterstrabanten-Verfinsterungen. 1830.

Beobachtet auf der Prager Sternwarte.

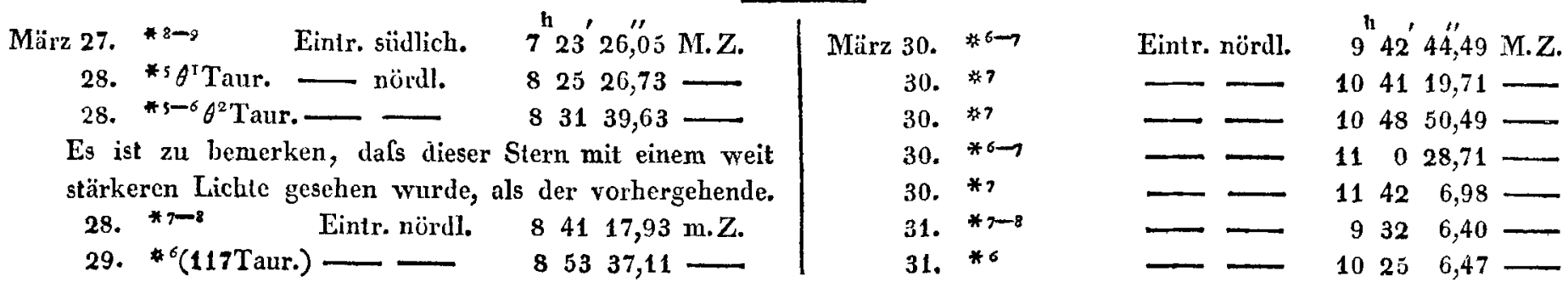


April 5. *4 (rLeon.) Einir. suidlich

h ' '

26.

26. *8-9

26. और

26. * 9

26. $\star 9$

26. * $* 0$

$26 . \$ 9$

26.

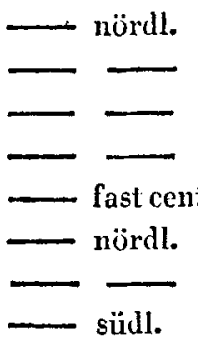

82623 ,18 M.Z.

$848 \quad 2,38$

$8 \quad 54 \quad 6,28$

$\begin{array}{lll}9 & 31 & 2,38\end{array}$

$\begin{array}{lll}9 & 38 & 46,59\end{array}$

$\begin{array}{lll}10 & 0 & 19,70\end{array}$

10738,10

$1024 \quad 42,80$

$1034 \quad 3,31$
April 28. *

28. * (1Cancr.)
30. $8-8$
30. *7
1. *7-8

Eintr. nördl.

$8^{\text {h }} 20^{\prime} 5,15$ M.Z.

82217,75

101135,97

- siidl.

$1023 \quad 20,76$

May 1. *7-8

- südl.

$10 \quad 13 \quad 37,11$

5.
Jupiterstrabanten

May 4. Eintrilt II. $13^{\text {h }} 49^{\prime} 34,49$ M.Z. Streifen deutlich. 7.

Hallaschka.

Schreiben des Herrn Professors Schwerd, Directors der Sternwarte in Speicr, an den Herausgeber. Speier 1830. Juni 2.

Ich iibergebe lbnen hier meine bisherigen Beobachtungen des jetzt sichtbaren Kometen.

\begin{tabular}{|c|c|c|c|c|c|c|c|c|c|c|}
\hline \multirow{2}{*}{\multicolumn{2}{|c|}{$\underbrace{\begin{array}{c}\text { Mittlere Zeit } \\
\text { in Speier. }\end{array}}$}} & \multirow{3}{*}{$\underbrace{\begin{array}{c}\text { Verglichene } \\
\text { Sterne. }\end{array}}_{\text {H.C.p.188. } 211514^{\prime}}$} & \multicolumn{3}{|c|}{ Ascensio recta apparens } & \multirow{3}{*}{$\underbrace{\begin{array}{c}\text { Anzahl } \\
\text { der } \\
\text { Vergl. }\end{array}}_{2}$} & \multicolumn{3}{|c|}{ Declinatio apparens } & \multirow{2}{*}{$\begin{array}{c}\text { Anzahl } \\
\text { der } \\
\text { Vergl. }\end{array}$} \\
\hline & & & & & & & Stern & & & \\
\hline May 4 & $1535^{\prime} 26^{\prime \prime}$ & & 3191832 & -2242 & $318 \stackrel{\circ}{55} 50$ & & $+17^{\circ} 44^{\prime} \mathbf{3}^{\prime}$ & +1547 & +175950 & 2 \\
\hline 5 & 15236 & --211031 & 318749 & +5327 & 319116 & 5 & +183025 & -225 & +18280 & 5 \\
\hline & 145855 & $-\square 211633$ & 3193824 & -377 & $319 \quad 117$ & 3 & & & & \\
\hline 6 & 145213 & H.C.p. 32. 21751 & $317 \quad 058$ & $+2^{\circ} 515$ & 319613 & 1 & +19036 & -546 & +185450 & $\mathbf{1}$ \\
\hline & 145213 & e Pegasi & 3183330 & +3239 & $319 \quad 6 \quad 9$ & 1 & & & & \\
\hline 17 & 133744 & fPegasi & $32034 \quad 4$ & -5828 & 3193536 & 6 & +225353 & +137 & +225530 & 6 \\
\hline 20 & 12350 & H.C.p. 29. 211552 & 3192447 & +827 & 3193314 & 7 & +234549 & -016 & +234533 & 7 \\
\hline & 122316 & $-\longrightarrow 211459$ & 3191138 & +2142 & 3193320 & 3 & +23489 & -243 & +233626 & 3 \\
\hline & 11429 & . XXI. 120 & 3191545 & -632 & $319 \quad 913$ & 6 & +252651 & -44 & +252247 & 6 \\
\hline & $12 \quad 224$ & derselbe & 3191545 & - 646 & 319859 & 2 & & & & \\
\hline
\end{tabular}

Ans den Beobachtungen rom 5., 17. und 27. May habe ich die folgenden Elemente berechnet, welche die mittlere Beobachlung in Länge und Breile bis auf $11^{\prime \prime}$ und $18^{\prime \prime}$ dărstellen.

Zeit des Perihels 1830 April 9,034455 mittl. Speierer Zeit

$$
\begin{aligned}
& \text { Lünge . . . } 212^{\circ} 1^{\prime} 13^{\prime \prime} \\
& \Omega \quad \text {. . . } 2061814 \\
& \text { Neigung . . . } 212044 \\
& \text { log. q. . . . . 9,96392 }
\end{aligned}
$$

\begin{tabular}{|c|c|c|c|}
\hline \multicolumn{3}{|c|}{ Geoc, Breite. } & Lic \\
\hline & $77^{\circ} 40$ & $25^{\prime \prime}$ & 1 \\
\hline & 4414 & 10 & \\
\hline & 0 & 0 & 3 \\
\hline & 2351 & 8 & 9,5 \\
\hline & 3233 & 40 & \\
\hline & 3630 & 25 & 2,1 \\
\hline & 3830 & 10 & \\
\hline & 4254 & 2 & 0,4 \\
\hline
\end{tabular}

Aus dicsen Elementen ergeben sich die nachstehenden Positionen und Lichtstärken:

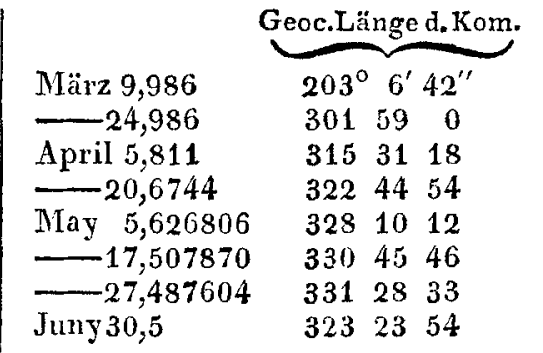

Der Komet mufs demnach schon in den ersten Tagen des Miärz den Bewohnern der südlichon Hemisphäre in dem Sternbilde des Schiffs sichtbar geworden eeyn, und, nachdem $\mathrm{er}$ am Südpol vorübergegangen, gegen den $25^{\text {sten }}$ desselben Monats zwischen den Sternbildern des Indianers und des Kranichs in seinem stärksten Glanze sich gezeigt haben. Schwerd.

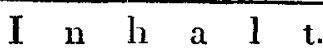

Aus einem Schreiben des Herrn Doctors u. Ritters Olbers. p. 285. - Beobl. auf der Prager Sternwarte des 1830 den 21 . April zu Marseille von Ganbart entdeckten Kometen. p. 285. - Ueber einige neulich beobachtete Anomalien der Pistorschen Heberbarometer von Herrn Prof. $\nu_{0}$ Riese in Bonn. p. 287. - Mondsdistanzen in Sibirien. p. 277. - Sternbedeckungen vom Monde und Jupiterstrabanten-Verfinsterungen, beobachtet auf der Prager Sternwarte. p. 281 . - Schreiben des Herrn Professors Schwerd an den Herausgeber. p. 299. 